\title{
Lost in translation: neuroscience and the public
}

\author{
Julie M. Robillard and Judy Illes
}

The Nature Reviews Neuroscience Science and Society article by Illes et al. (Neurotalk: improving the communication of neuroscience research, Nature Reviews Neuroscience 11, 61-69 (2010) $)^{1}$ highlights the need for improved dialogue between neuroscientists and public, and suggests that empirical research on neuroscience communication is an essential start. Keehner and Fischer (Naive realism in public perceptions of neuroimages, Nature Reviews Neuroscience 12 Jan 2011(doi:10.1038/nrn2773-c1)) ${ }^{2}$ respond to this call with an analysis of the 'dazzle effect' of neuroimages. Like Racine et al. ${ }^{3}$, they find that neurorealism plays a part in the credibility and seductive nature of brain images. Keehner and Fischer stress the importance of choosing visualization techniques with care, keeping in mind the impact that resulting images have on public perception. Communicating results meaningfully is a key goal for the neuroscience community.

To understand the scope of the challenge, we conducted a pilot study of the experience of neuroscientists in communicating their results. We surveyed a convenience sample of 600 neuroscientists whose work spans the whole academic and scientific range of the professional community. Of the 73 completed responses, 24 were from students (33\%), 12 from postdoctoral fellows (16\%), 28 from faculty (38\%) and 9 from technologists and other staff (12\%). Of the entire responder cohort, $19 \%$ reported that advances in brain research are well (16\%) or very well (3\%) communicated to the public. By contrast, $34 \%$ of responders reported that advances in brain research are poorly (29\%) or very poorly (5\%) communicated. A majority of responders (65\%) reported that they interact with journalists to communicate information about their research less than once per year. Nearly half (44\%) answered that their efforts to communicate about their research to the public are not valued by their institution.

Eleven responders augmented the quantitative data with qualitative responses. The theme of animals in research as a considerable barrier to communicating about neuroscience appeared in three responses. For example, one person commented that, "because I work with rats, I always feel on my guard whenever I speak to journalists because [institution] has made it clear that they want to avoid any controversy regarding the utility of animal research". Other barriers cited include lack of resources, as demonstrated by this comment: "I rarely communicate with the general public about my research; this is something I feel I should do more of but I'm not sure how to go about it". The need to simplify information and mitigate sensationalism in the press was another major theme in the open-ended responses. One respondent wrote: "research tends to be oversimplified and sensationalized ... but 'real' science probably doesn't sell". The attribution of the primary cause of poor neuroscience communication was divided however. Two comments suggested that scientists are the source: "a lot of researchers tend to overstate the significance of their data". Other respondents suggested that journalists are the source: "most of our findings get lost in translation".

Taken together, our data, those of Keehner and Fischer on neuroimages and data from others ${ }^{4-7}$ strongly support the need for a comprehensive and large-scale evaluation of the current state of neuroscience communication. The development of evidence-based recommendations is crucial to promote innovative strategies for mutually-informed, multidirectional engagement.
Judy IIles and Julie M. Robillard are at the National Core for Neuroethics, the University of British Columbia, 2211 Wesbrook Mall, Koerner S124, Vancouver, BC V6T 2B5, Canada. Correspondence to J.I. e-mail:jilles@interchange.ubc.ca doi: 10.1038/nrn2773-c2 Published online 12 January 2011

1. Illes, J. et al. Neurotalk: improving the communication of neuroscience research. Nature Rev. Neurosci. 11, 61-69 (2010).

2. Keehner, M. \& Fischer, M. H. Naive realism in public perceptions of neuroimages. Nature Rev. Neurosci. 12 Jan 2011 (doi:10.1038/nrn2773-c1).

3. Racine, E., Bar-llan, O. $\&$ Illes, J. fMRI in the public eye. Nature Rev. Neurosci. 6, 159-164 (2005).

4. Racine, E., Waldman, S., Rosenberg, J. \& Illes, J. Contemporary neuroscience in the media. Soc. Sci. Med. 71, 725-733 (2010).

5. Morein-Zamir, S. \& Sahakian, B. J. Neuroethics and public engagement training needed for neuroscientists. Trends Cogn. Sci. 14, 49-51 (2010).

6. Friedman, D. P. Public outreach: a scientific imperative. J. Neurosci. 28, 11743-11745 (2008).

Bubela, T. et al. Science communication reconsidered. Nature Biotechnol. 27, 514-518 (2009). 